\title{
KLOE measurements of $\mathrm{K}_{\mathrm{L}}$ lifetime and absolute branching ratio of $K^{+} \rightarrow \pi^{+} \pi^{-} \pi^{+}(\gamma)$
}

\section{Patrizia de Simone on behalf of the KLOE collaboration* ${ }^{\dagger}$}

Laboratori Nazionali di Frascati, I.N.F.N., Via E.Fermi 40, Frascati (Rome), Italy

E-mail: patrizia.desimonedlnf.infn.it

The KLOE experiment at the Frascati $e^{+} e^{-}$collider DAФNE, operating at the $\phi$ peak, provides unique opportunities for precision measurements of kaon properties. We present the preliminary update of the $\mathrm{K}_{\mathrm{L}}$ lifetime measurement, $\tau_{L}=50.56 \pm 0.14_{\text {stat }} \pm 0.21_{\text {syst }}$ ns, from a sample of $\sim 46$ million $\mathrm{K}_{\mathrm{L}} \rightarrow \pi^{0} \pi^{0} \pi^{0}$ events, tagged by $\mathrm{K}_{\mathrm{S}} \rightarrow \pi^{+} \pi^{-}$decays. The present error on the $\mathrm{K}_{\mathrm{L}}$ lifetime still limits the accuracy of the determination of the $V_{u s}$ CKM matrix element. We also discuss studies of the branching ratio for $\mathrm{K}^{+} \rightarrow \pi^{+} \pi^{-} \pi^{+}(\gamma)$ using approximately $10 \%$ of all KLOE data. This measurement would supersede the previous old result dating back to 1972 and would complete the KLOE program of precise and fully inclusive measurements of the $\mathrm{K}^{ \pm}$dominant branching ratios.

European Physical Society Europhysics Conference on High Energy Physics, EPS-HEP 2009, July 16 - 222009

Krakow, Poland

\section{*Speaker.}

${ }^{\dagger}$ F. Ambrosino, A. Antonelli, M. Antonelli, F. Archilli, P. Beltrame, G. Bencivenni, C. Bini, C. Bloise, S. Bocchetta, F. Bossi, P. Branchini, G. Capon, D. Capriotti, T. Capussela, F. Ceradini, P. Ciambrone, E. De Lucia, A. De Santis, P. De Simone, G. De Zorzi, A. Denig, A. Di Domenico, C. Di Donato, B. Di Micco, M. Dreucci, G. Felici, S. Fiore, P. Franzini, C. Gatti, P. Gauzzi, S. Giovannella, E. Graziani, M. Jacewicz, V. Kulikov, G. Lanfranchi, J. Lee-Franzini, M. Martini, P. Massarotti, S. Meola, S. Miscetti, M. Moulson, S. Müller, F. Murtas, M. Napolitano, F. Nguyen, M. Palutan, A. Passeri, V. Patera, P. Santangelo, B. Sciascia, A. Sibidanov, T. Spadaro, L. Tortora, P. Valente, G. Venanzoni, R. Versaci. 


\section{The KLOE experiment at DA $\Phi N E$}

DA $\Phi$ NE, the Frascati $\phi-$ factory[1], is an $e^{+} e^{-}$collider working at a center of mass energy of $\sqrt{s} \simeq m_{\phi} \simeq 1020 \mathrm{MeV}$. The $\phi$ production cross section is $\sim 3 \mu \mathrm{b}$. The beams collide at the interaction point (IP) with a crossing angle $\theta_{x} \simeq 25 \mathrm{mrad}$, therefore the $\phi$ 's are produced with a small momentum of $\sim 12.5 \mathrm{MeV}$ in the horizontal plane, and decay in almost collinear and monochromatic neutral (34\%) and charged (49\%) kaon pairs.

The KLOE detector consists of a large volume drift chamber surrounded by an electromagnetic calorimeter. A superconducting coil provides a $0.52 \mathrm{~T}$ solenoidal magnetic field. The tracking detector is a cylindrical drift chamber [2] (DC) $4 \mathrm{~m}$ diameter and $3.3 \mathrm{~m}$ long, with a total of $\sim 52000$ wires, of which $\sim 12000$ are sense wires. In order to minimize the multiple scattering and the $K_{L}$ regeneration, and to maximize the detection efficiency of low energy $\gamma$ 's, the DC works with an helium based gas mixture and its walls are made of light materials (mostly carbon fiber composites). The momentum resolution for tracks produced at large polar angle is $\sigma_{p \perp} / p_{\perp} \leq$ $0.4 \%$. Charged particle vertices are reconstructed with a spatial resolution of $\sim 3 \mathrm{~mm}$ [2]. The fine sampling lead-scintillating fiber calorimeter [2] (EMC) consists of a barrel and two end-caps, and has solid angle coverage of $98 \%$. Photon energies and arrival times are measured with resolutions $\sigma_{E} / E=5.7 \% / \sqrt{E(\mathrm{GeV})}$ and $\sigma_{t}=57 \mathrm{ps} / \sqrt{E(\mathrm{GeV})} \oplus 100 \mathrm{ps}$, respectively. Photon entry points are determined with an accuracy $\sigma_{z} \sim 1 \mathrm{~cm} / \sqrt{E(\mathrm{GeV})}$ along the fibers and $\sigma_{\perp} \sim 1 \mathrm{~cm}$ in the transverse direction.

The unique feature of a $\phi$-factory is the possibility of tagging. Specifically the detection of a $\mathrm{K}_{\mathrm{L}}\left(\mathrm{K}_{\mathrm{S}}\right)$ guarantees the presence of a $\mathrm{K}_{\mathrm{S}}\left(\mathrm{K}_{\mathrm{L}}\right)$ with known momentum and direction, the same holds for charged kaons pairs [2]. The availability of tagged kaons enables the precision measurement of absolute BR's. The $\mathrm{K}_{\mathrm{L}}$ beam is identified by the presence of a decay $\mathrm{K}_{\mathrm{S}} \rightarrow \pi^{+} \pi^{-}$. The $\mathrm{K}_{\mathrm{L}}$ momentum is given by the decay kinematics of $\phi \rightarrow \mathrm{K}_{\mathrm{L}} \mathrm{K}_{\mathrm{S}}$ using the reconstructed $\mathrm{K}_{\mathrm{S}}$ direction and the small velocity $v_{\phi}$ of the $\phi\left(v_{\phi}\right.$ reconstructed run by run with the Bhabha events). The selection of the $\mathrm{K}^{ \pm}$beam is done reconstructing the 2 body decays $\mathrm{K}^{ \pm} \rightarrow \pi^{ \pm} \pi^{o}$ and $\mathrm{K}^{ \pm} \rightarrow \mu^{ \pm} \bar{v}(v)$. These decays are identified from two clear peaks in the momentum of the charged secondary tracks in the kaon rest frame.

KLOE completed the data taking in March 2006 with a total integrated luminosity of $~$ $2.5 \mathrm{fb}^{-1}$, corresponding to $\sim 7.5 \times 10^{9} \phi$-mesons produced.

\section{2. $\mathrm{K}_{\mathrm{L}}$ lifetime}

Two different measurements of the $\mathrm{K}_{\mathrm{L}}$ lifetime, $\tau_{L}$, has been already published by KLOE. The first one, $\tau_{L}=50.92 \pm 0.17_{\text {stat }} \pm 0.25_{\text {syst }} \mathrm{ns}$ [3], has been obtained from the fit of the proper time distribution of $\sim 8.5 \times 10^{6} \mathrm{~K}_{\mathrm{L}} \rightarrow 3 \pi^{0}$ decays tagged by $\mathrm{K}_{\mathrm{S}} \rightarrow \pi^{+} \pi^{-}$, while the second one, $\tau_{L}=$ $50.72 \pm 0.11_{\text {stat }} \pm 0.35_{\text {syst }}$ ns [4], by counting the $\mathrm{K}_{\mathrm{L}}$ decays and imposing the constrain $\Sigma B R\left(K_{L}\right)=1$, on a sample of $\sim 13 \times 10^{6}$ tagged $\mathrm{K}_{\mathrm{L}}$. However, because the error on $\tau_{L}$ is the main limiting factor on the accuracy of $V_{u s}$ from the $\mathrm{K}_{\mathrm{L}}$ semileptonic decays [5], we are analyzing the whole KLOE data sample to improve both the statistical and systematic accuracies on the $\tau_{L}$ measurement. We present here the preliminary result based on $\sim 2 / 3$ of the KLOE data sample. 
The timing performance of the EMC allows to measure the flight path of the $\mathrm{K}_{\mathrm{L}} \rightarrow$ neutrals, with a method that is unique: for each $\gamma$, the EMC measures the arrival time and the conversion point, while $\mathrm{K}_{\mathrm{L}}$ direction and momentum are obtained with $\mathrm{DC}$ measurements, see fig. 1 (left). To obtain an high and uniform $\mathrm{K}_{\mathrm{L}} \rightarrow \pi^{o} \pi^{o} \pi^{o}$ vertex reconstruction efficiency, we require at least 3 $\gamma$ 's, see figure 1 (right).
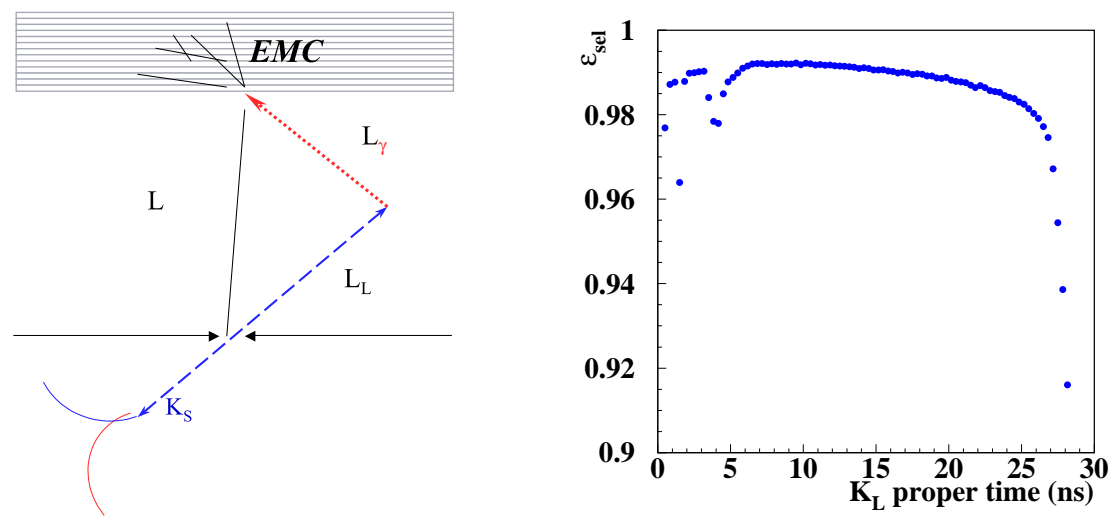

Figure 1: Left: $K_{L}$ flight path measurement. Right: signal selection efficiency (MC distribution).

A data sample of selected $\mathrm{K}_{\mathrm{L}} \rightarrow \pi^{ \pm} \pi^{\mp} \pi^{o}$ decays, has been used to study the neutral vertex reconstruction quality:

1. the accuracy of the $\mathrm{K}_{\mathrm{L}}$ flight path measurement with a single $\gamma$ is checked by comparing it with the decay vertex position obtained with DC tracking measurements. The spatial resolution of the decay vertex is $\sigma\left(\rho_{v t x}\right) \sim 2.2 \mathrm{~cm}$;

2. the single $\gamma$ reconstruction efficiency has been measured, $\varepsilon_{\gamma}=N_{\gamma \text {, rec }} / N_{\gamma \text {, tag }}$, where $N_{\gamma}$, tag is the number of events where a vertex $\pi^{+} \pi^{-} \gamma$ has been reconstructed, and $N_{\gamma \text {, rec }}$ is the number of events in which a second $\gamma$ is detected where we expect from the kinematics of the $\mathrm{K}_{\mathrm{L}} \rightarrow \pi^{ \pm} \pi^{\mp} \pi^{o}$ decay. The Monte Carlo has been corrected with the ratio $\varepsilon_{\text {data }} / \varepsilon_{M C}$ using a hit-or-miss method.

The proper time, $t^{*}$, distribution obtained from the selected sample of $\mathrm{K}_{\mathrm{L}} \rightarrow \pi^{o} \pi^{o} \pi^{o}$ is shown in fig. 2 (left) together with the fit result (the red solid line) in the range $8<t^{*}<26 \mathrm{~ns}$. The blue region represents the $\sim 1.81 \%$ residual background shape: the two peaks before $8 \mathrm{~ns}$ are due to regeneration events and nuclear interactions on the beam pipe and on the internal wall of the DC, while after $25 \mathrm{~ns}$ it is due mainly to the $\mathrm{K}_{\mathrm{L}} \rightarrow \pi^{ \pm} \pi^{\mp} \pi^{o}$ decays. The preliminary result is:

$$
\tau_{L}=50.56 \pm 0.14_{\text {stat }} \pm 0.21_{\text {syst }} \mathrm{ns}=50.56 \pm 0.25 \mathrm{~ns}
$$

compatible with the previous KLOE [3], [4] measurements, still to be much improved. The statistical error can be improved by decreasing the lower limit of the fit region, properly accounting for the $\mathrm{K}_{\mathrm{L}}$ beam losses on the regenerating surfaces. Moreover, the statistical error on $\tau_{L}$ is expected to further decrease to $\sim 0.1 \mathrm{~ns}$, once we will complete the analysis on all KLOE data. 


\section{Absolute $\mathrm{K}^{+} \rightarrow \pi^{+} \pi^{-} \pi^{+}(\gamma)$ branching ratio studies.}

The measurement of the BR of the decay completes the KLOE program of precise and fully inclusive kaon dominant BR's measurements. The most recent result, $\operatorname{BR}\left(K^{ \pm} \rightarrow \pi^{ \pm} \pi^{+} \pi^{-}=\right.$ $(5.56 \pm 0.20) \%$ ) [6], dates back to more than 30 years ago.

We plan to use two normalization samples given by the two tags $K_{\mu 2}$ and $K_{\pi 2}$. The track of the tagged kaon is backward extrapolated to the interacion point, then the kinematic of the decay $\phi \rightarrow K^{+} K^{-}$, give us the possibility to define direction and momentum of the signal kaon.

The decay products of the kaons have a very low momentum, less than $200 \mathrm{MeV} / \mathrm{c}$, and curl up in the KLOE magnetic field; this increase the probability to reconstruct broken tracks and fake vertices. If we select kaon decays within the inner volume of the DC, the maximum number of tracks that must be reconstructed is three instead of four, and the quality of the reconstruction increases. We require at least two reconstructed tracks in the DC (pion candidates), then if their backward extrapolations do a vertex with the path of the signal kaon before the inner wall of the DC (the geometrical acceptance is $\sim 26 \%$ ), we evaluate the missing mass of the decay. The fig. 2 (right) showes the comparison between data and $\mathrm{MC}$ of the missing mass spectrum, that has a peak centered around the mass of the pion, the MC background contribution is superimposed.
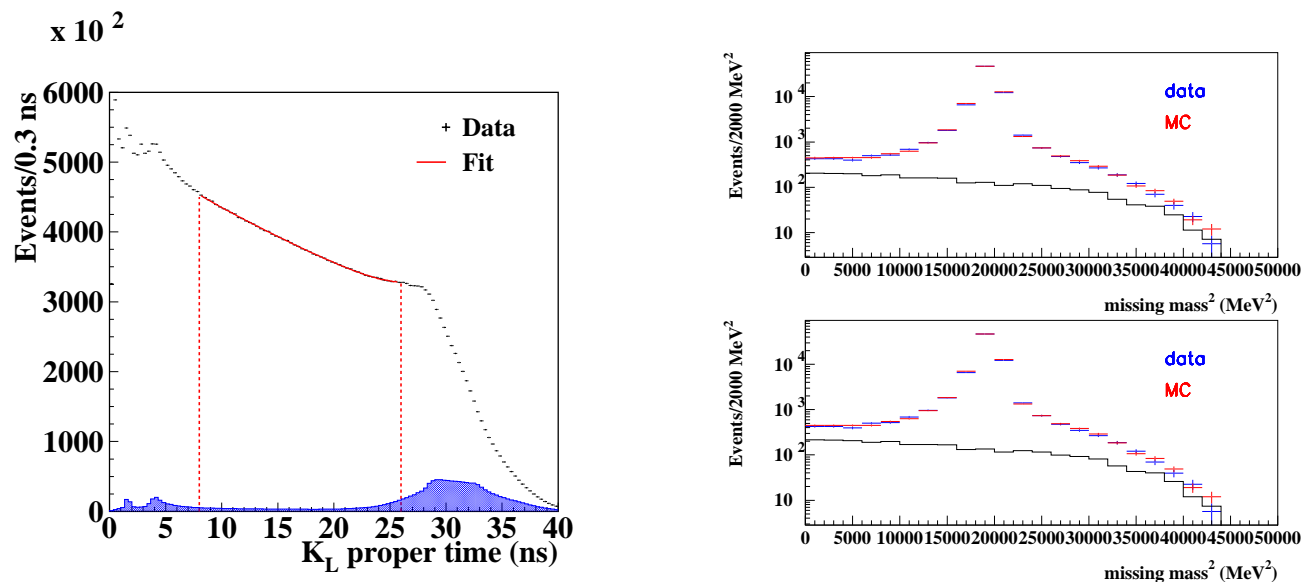

Figure 2: Left: fit to the $\mathrm{K}_{\mathrm{L}}$ proper time distribution in the range 8-26 ns, the fit is shown as the solid red line, and the background shape from MC is the blue region. Right: squared missing mass distribution for $\mu v$ tag (top) and $\pi \pi^{0}$ tag (bottom). In black the background, from MC.

The selection efficiency will be evaluated from MC, and corrections will be applied to account for data-MC differences in tracking. The measurement, still in progress, can reach a statistical relative error of a few $\times 0.1 \%$.

\section{References}

[1] A. Drago, et al. LNF-03/012 (2003).

[2] F. Bossi, et al., Precision Kaon and Hadron Physics with KLOE, La Rivista del Nuovo Cimento, Vol.31, serie 5, N.10, (2008). 
[3] KLOE coll., F.Ambrosino, et al., Phys. Lett. B 626, 15 (2005).

[4] KLOE coll., F.Ambrosino, et al., Phys. Lett. B 632, 43 (2006).

[5] KLOE coll., F.Ambrosino, et al., JHEP 04, 059 (2008).

[6] I.H. Chiang et al., Phys. Rev. D 6, 1972 (1254). 